\title{
Transient vibration analysis of a completely free plate using modes obtained by Gorman's Superposition Method
}

\author{
Y Mochida*, S Ilanko \\ Department of Engineering, The University of Waikato, Te Whare Wananga o Waikato, Hamilton, New Zealand
}

\begin{abstract}
This paper shows that the transient response of a plate undergoing flexural vibration can be calculated accurately and efficiently using the natural frequencies and modes obtained from the Superposition Method. The response of a completely free plate is used to demonstrate this. The case considered is one where all supports of a simply supported thin rectangular plate under self weight are suddenly removed. The resulting motion consists of a combination of the natural modes of a completely free plate. The modal superposition method is used for determining the transient response, and the natural frequencies and mode shapes of the plates used are obtained by Gorman's Superposition Method. These are compared with corresponding results based on the modes using the Rayleigh-Ritz method using the ordinary and degenerated free-free beam functions. There is an excellent agreement between the results from both approaches but the Superposition Method has shown faster convergence and the results may serve as benchmarks for the transient response of completely free plates.
\end{abstract}

$\begin{array}{llll}\text { Nomenclature } & W & \begin{array}{l}\text { plate lateral deflection } \\ \text { plate spatial co-ordinates }\end{array} \\ a & \text { plate dimension in } x \text { direction } & x, y & \begin{array}{l}\text { Poisson's ratio of material } \\ \text { density of plate }\end{array} \\ b & \text { plate dimension in } y \text { direction } & \rho & \text { aspect ratio of plate } b / a \\ D & \text { plate flexural rigidity, }\left(E h^{3} / 12\right) /\left(1-v^{2}\right) & \Phi & \text { radian frequency of vibration } \\ E & \text { elastic modulus of the material } & \omega & \\ h & \text { thickness of plate } & & \\ w & \text { transient response of plate } & & \end{array}$

Keywords: transient vibration, plates, Superposition, Rayleigh-Ritz method, W-W algorithm 


\section{Introduction}

Thin plates are one of the most common components in machines and structures, and there is a vast literature devoted to estimate the natural frequencies of thin plates. An excellent review of the literature relating to vibration analysis of plates was published by Leissa [1]. Among the methods utilised for vibration analysis, it is known that Gorman's Superposition method is very efficient and accurate for a range of geometric shapes and in many cases the results from the Superposition method are the benchmarks for the natural frequencies [2-6]. In a recent publication, the Superposition Method was shown to be applicable for the determination of steady state response of plates [7]. In this paper we show it can also be used to accurately and efficiently determine the transient response of an undamped plate undergoing flexural vibration.

Research into analysis of the transient response of plates has spanned several decades. In one of the earliest papers on this subject, Forsyth and Warburton [8] predicted the transient response of cantilever plates to the impulse load using the natural frequencies and mode shapes obtained by applying the Rayleigh method. Craggs [9] solved the transient problems of simply supported, clamped and cantilever plates using the transition matrix method. Nagaya [10] investigated the transient response of a continuous plate on elastic supports to an impact load using the Laplace transform method. The Finite Element Method was used by Rock and Hinton [11] to obtain the transient response of both simply supported thick and thin plates. Coleby and Mazumdar [12] analysed the large amplitude transient response of an elliptical plate using the Berger method. Celep [13] presented the transient response of a thin elastic plate supported on a foundation that reacts in compression only, where the plate displacement is approximated by the product of vibration modes of the free beam. Nath and Shukala [14] have carried out the non-linear transient analysis of moderately thick laminated composite plates with mix of clamped, simply supported, and free boundary conditions based on Chebyshev approximation. In a recent publication, Abrate [15] examined the transient response of beams, plates and shells to certain pulse type loads using the modal expansion technique. The above list is not complete but to our knowledge, no results have been reported for the transient vibration of completely free plates, based on modes obtained using the Superposition Method. We believe this paper will complement the recent publication on steady state response by the Superposition Method.

In this paper, the transient response of a thin rectangular plate subject to an initial displacement corresponding to that of a plate under uniform load distribution with all edges simply supported is studied when all supports are suddenly removed. The plate after being released from its supports is treated as a completely free plate. The natural frequencies and mode shapes of the plates used in this 
study are obtained by the Superposition method (SM). The results are compared with the response computed based on the natural frequencies and mode shapes given by the Rayleigh-Ritz (R-R) method with the ordinary and degenerated free-free beam functions. The response generated from the $\mathrm{SM}$ agrees closely with the R-R results but it is noted that the SM method converges faster. For the same matrix size, the SM method gives more accurate results and we believe the results presented may be regarded as benchmarks for future comparisons.

\section{Procedure}

\subsection{Natural frequencies and modes of vibration}

Consider the motion of the completely free rectangular plate with the dimensions $a$ and $b$ as shown in Fig. 1. The natural frequencies and modes will be calculated using both the variational method based on an energy functional and the partial differential equation, i.e. the Rayleigh-Ritz Method and the Superposition Method. They are described in detail, in reference [1, 16] and [2, 3] respectively. The essential steps in the derivations are presented here for completeness.

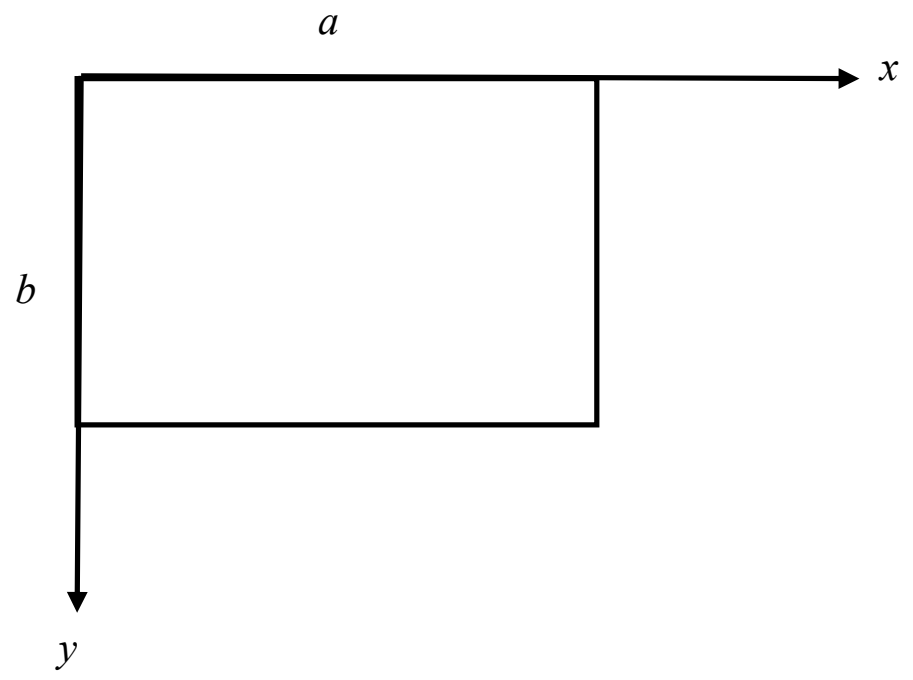

Fig. 1 A completely free plate 


\section{The Rayleigh-Ritz Method}

For the R-R method, it is assumed that the out-of-plane displacement, $W(x, y)$, is taken in the form of the following series,

$$
W(x, y)=\sum \sum G_{m n} X_{m}(x) Y_{n}(y) \quad m, n=1,2,3
$$

where $X_{m}(x)$ and $Y_{n}(y)$ are ordinary free-free beam functions, which are given below [1].

$$
\begin{gathered}
X_{1}(x)=1 \\
X_{2}(x)=1-\frac{2 x}{a} \\
X_{m}(x)=\cos \gamma_{1}\left(\frac{x}{a}-\frac{1}{2}\right)+\theta_{m 1} \cosh \gamma_{1}\left(\frac{x}{a}-\frac{1}{2}\right) \quad(m=3,5,7, \ldots)
\end{gathered}
$$

and

$$
X_{m}(x)=\sin \gamma_{2}\left(\frac{x}{a}-\frac{1}{2}\right)+\theta_{m 2} \sinh \gamma_{1}\left(\frac{x}{a}-\frac{1}{2}\right) \quad(m=4,6,8, \ldots)
$$

where $\theta_{m 1}=-\sin \left(\gamma_{1} / 2\right) / \sinh \left(\gamma_{1} / 2\right)$ and $\theta_{m 2}=\sin \left(\gamma_{2} / 2\right) / \sinh \left(\gamma_{2} / 2\right)$

The values of $\gamma_{1}$ and $\gamma_{2}$ are obtained as roots of following Eq. (6) and Eq. (7) respectively.

$$
\begin{aligned}
& \tan \left(\gamma_{1} / 2\right)+\tanh \left(\gamma_{1} / 2\right)=0 \\
& \tan \left(\gamma_{2} / 2\right)-\tanh \left(\gamma_{2} / 2\right)=0
\end{aligned}
$$

Using the R-R method brings a set of equations expressed as [16]

$$
\frac{\partial V_{\max }}{\partial G_{m n}}-\frac{\omega^{2} \rho h}{2} \frac{\partial}{\partial G_{m n}} \int_{0}^{a} \int_{0}^{b} W^{2} d x d y=0
$$

where $V_{\max }$ is maximum potential energy, which is given by 


$$
V_{\text {max }}=\frac{D}{2} \int_{0}^{a} \int_{0}^{b}\left[\left(\frac{\partial^{2} W}{\partial x^{2}}\right)^{2}+\left(\frac{\partial^{2} W}{\partial y^{2}}\right)^{2}+2 v \frac{\partial^{2} W}{\partial x^{2}} \frac{\partial^{2} W}{\partial y^{2}}+2(1-v)\left(\frac{\partial^{2} W}{\partial x \partial y}\right)\right] d x d y
$$

By inserting Eq. (9) and Eq. (1) into Eq. (8), one obtains a set of homogeneous linear algebraic equations in $G_{m n}$. The natural frequencies are determined by equating the determinant of the system to zero. The coefficients, $G$ 's, are given as the eigenvector corresponding to the natural frequency.

However, it has been noted that the ordinary free-free beam functions do not completely satisfy the free edge condition of plates because of over-constraining of the boundaries [17]. Bassily and Dickinson introduce the concept of degenerated beam functions to relax the end conditions in [17]. This relaxation can be achieved by simply floating $\theta$ in Eqs (4) and (5). The $\theta$ is replaced by a coefficient, which will be determined during the usual minimisation procedure in the R-R method.

\section{The Superposition Method}

Determining the natural frequencies and mode shapes of the completely free plates by using the SM is described in detail by Gorman [2, 3]. In this paper, the SM is utilised and the W-W algorithm is used to determine the natural frequencies to ensure double roots of the frequency equation are not missed as explained later.

The partial differential equation governing the out-of-plane vibration of rectangular plates, is expressed in non-dimensional form using dimensionless coordinates $\xi$ and $\eta$, where $\xi=x / a, \eta=y / b$, The equation is written as

$$
\frac{\partial^{4} W(\xi, \eta)}{\partial \eta^{4}}+2 \Phi^{2} \frac{\partial^{4} W(\xi, \eta)}{\partial \eta^{2} \partial \xi^{2}}+\Phi^{4}\left\{\frac{\partial^{4} W(\xi, \eta)}{\partial \xi^{4}}-\lambda^{4} W(\xi, \eta)\right\}=0
$$

where $\lambda^{2}=\omega a^{2} \sqrt{\rho h / D}$ and $\Phi$ is the plate aspect ratio $b / a$.

In the $\mathrm{SM}$, the plate is considered $[2,3]$ as consisting of four plate problems which have exact solutions as shown in Fig. 2, and the displacement of the original plate, $W(x, y)$, is expressed as the sum of the displacement of the sub-systems which are referred to as the building blocks (Eq. (12)).

$$
W(\xi, \eta)=W_{m}+W_{n}+W_{p}+W_{q}
$$




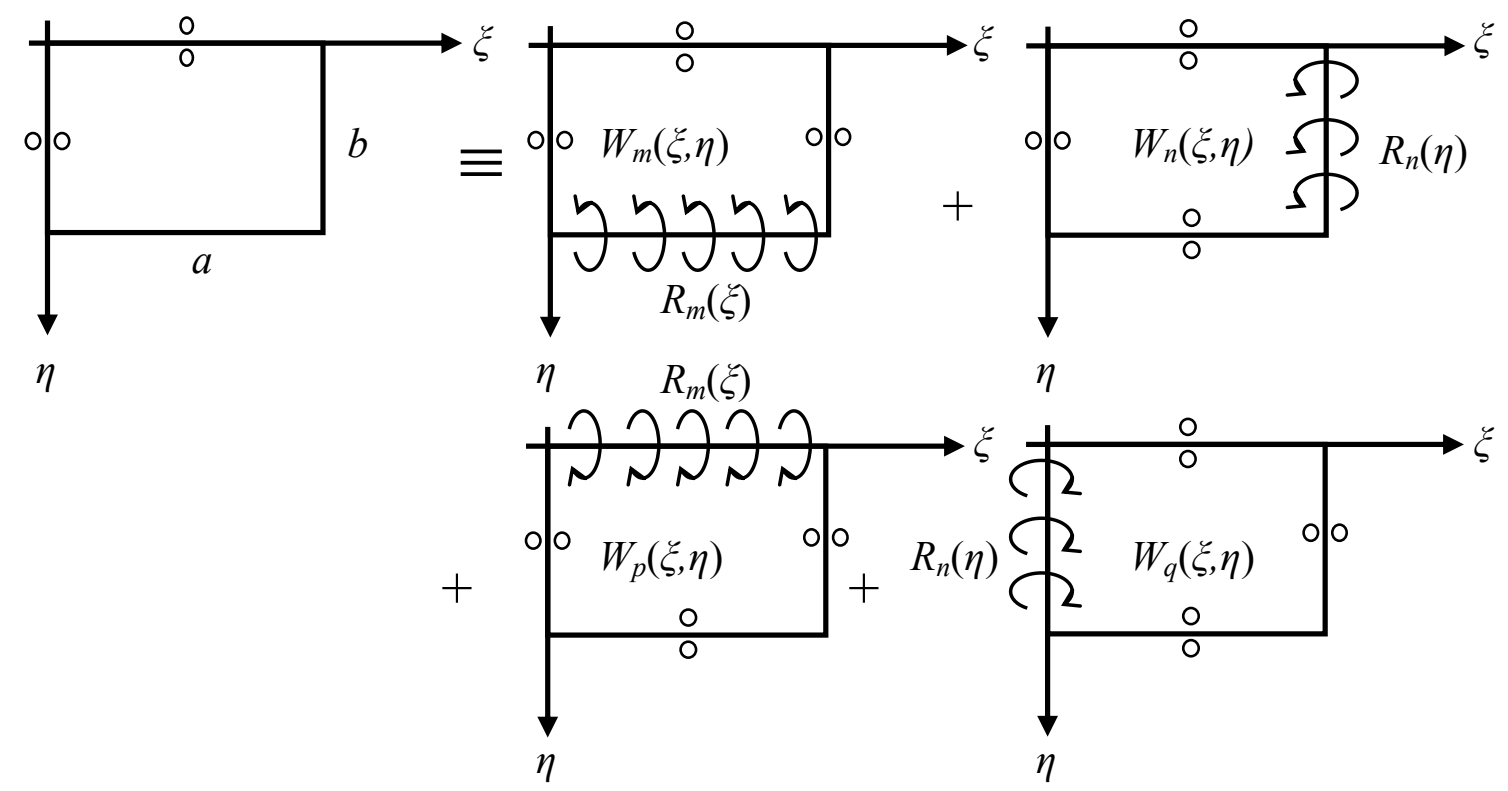

Fig. 2 Building blocks used to analyse the completely free plate

The displacements of the first building block is taken form of Lévy type solution,

$$
W_{m}(\xi, \eta)=\sum_{m=0,1 \cdots}^{k} Y_{m}(\eta) \cos m \pi \xi
$$

The edge rotation along the edge $\eta=1$ is expressed as following Fourier expansion

$$
\frac{\partial W_{m}(\xi, \eta)}{\partial \eta}=\sum_{m=0,1 \ldots}^{k} E_{m} \cos m \pi \xi
$$

By enforcing the boundary condition of zero vertical edge reaction and the equilibrium of edge rotation, the analytical function $Y_{m}(\eta)$ is readily determined. The solutions for $Y_{m}(\eta)$ are expressed in terms of the coefficients $E_{m}$, in reference [3] as

for $\lambda^{2}>(m \pi)^{2}$

$$
Y_{m}(\eta)=E_{m}\left(\theta_{m 11} \cosh \beta_{m} \eta+\theta_{m 12} \cos \gamma_{m} \eta\right)
$$

and, for $\lambda^{2}<(m \pi)^{2}$ 


$$
Y_{m}(\eta)=E_{m}\left(\theta_{m 21} \cosh \beta_{m} \eta+\theta_{m 22} \cosh \gamma_{m} \eta\right)
$$

where

$$
\beta_{m}=\Phi \sqrt{\lambda^{2}+(m \pi)^{2}}
$$

and

$$
\gamma_{m}=\Phi \sqrt{\lambda^{2}-(m \pi)^{2}} \text { or } \gamma_{m}=\Phi \sqrt{(m \pi)^{2}-\lambda^{2}} \text {, whichever is real. }
$$

The quantities $\theta$ are constants to be determined. The solution to the second building block is easily obtained from the first building block by interchanging the variables $\eta$ and $\xi$, using the inverse of the aspect ratio and changing subscript $m$ to $n$. Once the solutions to the first and second building blocks are obtained, solutions to the third and forth building blocks are determined by simply replacing $\eta$ in the first building block solution to $1-\eta, \xi$ in the second building block solution to $1-\xi$, and changing subscripts to $p$ and $q$ respectively.

These building blocks are superimposed in order to solve the original plate problem. Applying the boundary conditions of original plate problem, i.e. zero bending moment at the edges, using $k$ terms in each building block, yields a set of $4 k$ homogeneous algebraic equations relating $4 k$ coefficients, $E_{m}, E_{n}, E_{p}$ and $E_{q}$ which can be expressed in matrix form as follows:

$$
[A]\left\{E_{x}\right\}=\{0\}
$$

where $[A]$ is $4 k \times 4 k$ matrix, $\left\{E_{x}\right\}$ is $4 k \times 1$ column vector of coefficients, $E_{m}, E_{n}, E_{p}$ and $E_{q}$.

The natural frequencies are determined by searching for the $\lambda$ values for which the determinant of the system vanishes by trial and error. Once the $\lambda$ values are found, the coefficients, $E$ 's, are found by substituting into equation (19) and these give the natural modes of the plate. However, this procedure also picks up some unexpected values when the determinant goes through a pole (case (b) in Fig. 3), and misses the values associated with coincident modes of a square plate (case(c)), i.e. the symmetric-antisymmetric modes and the antisymmetric-symmetric modes about $x$ and $y$ axes. To ensure that coincident roots are not missed, the W-W algorithm [18-20] has been used. The coefficient matrix generated with the trial value is modified to an upper triangular matrix according to the $\mathrm{W}-\mathrm{W}$ algorithm. The number of negatives along the diagonal of the matrix is counted for each trial frequency, and any change in this number indicates the number of new roots between the previous trial frequency and the current trial frequency. Hence, the natural frequency parameters $\lambda$ can be delimited in this way. In addition, if the number changes in two, then the value of $\lambda$ is 
considered as associating with a coincident mode. However, using this procedure still gives some unexpected values when the determinant changes sign through zero at the poles and the values of $\lambda$ corresponds to the eigenvalues of the square plate having all edges fully constrained against rotation (case (d)). Strictly speaking the application of the W-W algorithm requires the knowledge of the natural frequencies of the fully constrained plate and each time such frequencies are passed the change in the sign count should be decreased by the number of such frequencies. This was effectively achieved using the following procedure. The distances of the determinant from the zero line are stored and at a given trial frequency which gives a determinant $d_{0}$, the previous two determinants are examined when the number of negatives changed. Let the previous two values of determinant $d_{1}$ and $d_{2}$ as shown in Fig. 3. If $d_{1}$ is closer to the zero line than $d_{2}$, then $\lambda$ is counted as the value associating with a natural frequency or coincident natural frequencies (when change of the negatives is two), otherwise the value of $\lambda$ is ignored. This procedure has eliminated the false roots and leaving only the required set of natural frequency parameters. These results agree closely with those in the literature [2-6].

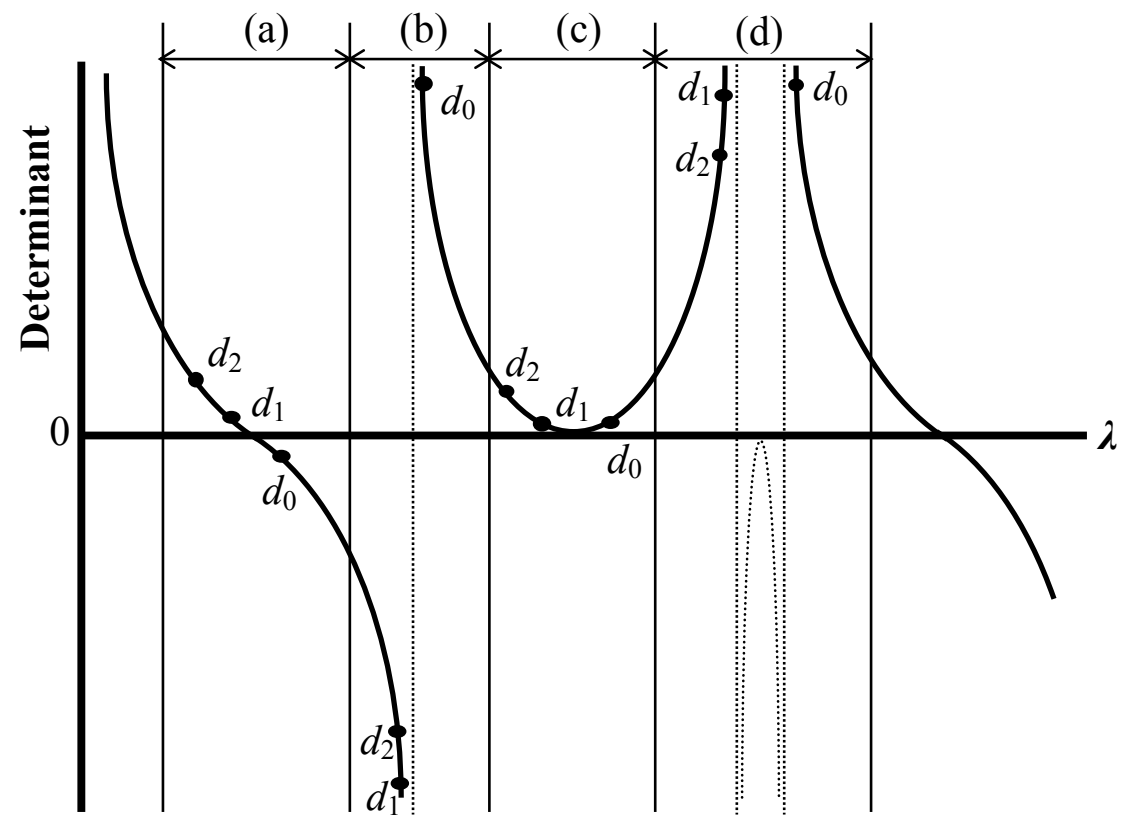

Fig. 3 Plot of the determinant vs. trial $\lambda$

\subsection{Transient vibrations}

In the transient analysis, the effect of damping has not been considered. The response due to an initial disturbance is then expressed in term of its modes as follows:

$$
w(x, y, t)=\sum a_{i} W_{i}(x, y) \sin \left(\omega_{i} t\right)+\sum b_{i} W_{i}(x, y) \cos \left(\omega_{i} t\right)
$$


where $W_{i}(x, y)$ is $\mathrm{i}^{\text {th }}$ normal mode and $\omega_{i}$ is the $\mathrm{i}^{\text {th }}$ natural frequency. $W_{i}(x, y)$ is given by Eq. (1) or (12). Eq. (20) has to satisfy the prescribed initial conditions, i.e. the displacement and/or velocity at $t=0$. An expression for the velocity may be obtained by differentiating Eq. (20) with respect to $t$.

$$
\dot{w}(x, y, t)=\sum \omega_{i}\left(a_{i} W_{i}(x, y) \cos \left(\omega_{i} t\right)-b_{i} W_{i} \sin \left(\omega_{i t}\right)\right)
$$

Let the displacement and the velocity at $t=0$ be following equations,

$$
w(x, y, 0)=w_{0}(x, y) \text { and } \dot{w}(x, y, 0)=\dot{w}_{0}(x, y)
$$

At $t=0$, Eq. (20) and (21) become,

$$
w_{0}(x, y)=\sum b_{i} W_{i}(x, y)
$$

and

$$
\dot{w}_{0}(x, y)=\sum \omega_{i} a_{i} W_{i}(x, y)
$$

The coefficients $a_{i}$ and $b_{i}$ may be determined by multiplying both sides of Eq. (23) and (24) by $W_{j}$ and integrating over the area of plate, i.e.,

$$
\iint w_{0}(x, y) W_{j}(x, y) d x d y=\iint \sum_{i} b_{i} W_{i}(x, y) W_{j}(x, y) d x d y
$$

and

$$
\iint \dot{w}_{0}(x, y) W_{j}(x, y) d x d y=\iint \sum_{i} \omega_{i} a_{i} W_{i}(x, y) W_{j}(x, y) d x d y
$$

However, from the orthogonality condition,

$$
\left.\iint W_{i}(x, y) W_{j}(x, y) d x d y=0 \quad \text { (if } i \neq j\right)
$$

From Eq. (25), Eq. (26) and (27), the coefficients $a_{i}$ and $b_{i}$ are given by,

$$
a_{j}=\frac{\iint \dot{w}_{0}(x, y) W_{j}(x, y) d x d y}{\omega_{i} \iint\left\{W_{j}(x, y)\right\}^{2} d x d y}
$$

and 


$$
b_{j}=\frac{\iint w_{0}(x, y) W_{j}(x, y) d x d y}{\iint\left\{W_{j}(x, y)\right\}^{2} d x d y}
$$

\subsection{Initial conditions}

The initial conditions given in this study are that there is an imposed deflection which is equal to that of a plate with all edges simply supported, and subject to a uniformly distributed load such as self weight and that the velocity is zero everywhere. The deflection expression for this case which is readily available in the literature [21] is,

$$
w_{0}(x, y)=\frac{16 q_{0}}{\pi^{6} D} \sum_{k} \sum_{l} \frac{\sin \frac{k \pi x}{a} \sin \frac{l \pi y}{b}}{k l\left(\frac{k^{2}}{a^{2}}+\frac{l^{2}}{b^{2}}\right)^{2}} \quad(k=1,3,5, \ldots l=1,3,5, \ldots)
$$

where $q_{o}$ is a load per area. The load is assumed as the weight of the plate per area, which is given by $q_{o}=\rho h g$. Five terms in each direction were found to be sufficient to ensure that the results for the displacement have converged to four significant figures, and therefore $k$ and $l$ in Eq (30) were set for nine in computation. It should be noted here that if the transient response of a plate that is freely falling under gravity after removal of its simple supports is required, the rigid body motion of the plate due to the gravity force $g t^{2} / 2$ should be added. The above initial condition was chosen as a convenient case to remember for the purpose of benchmarking and not due to any engineering significance.

\section{Result and discussion}

The transient response of the completely free plates has been calculated when the plates having all the edges simply supported are suddenly released from the all supports. The computation of the response of the plates for the various aspect ratios was done using the natural frequencies and modal shapes given by the Superposition method and the Rayleigh-Ritz method with the ordinary and degenerated free-free beam functions. The first fifty modes are used in the calculation. The displacement and time are given in dimensionless forms, which are $w /\left(\frac{16 q_{0} a^{4}}{\pi^{6} D}\right)$ and 
$t /\left(a^{2} \sqrt{\rho h / D}\right)$ respectively. All responses were calculated by using the software MATLAB in default double precision. However, the maximum number of terms used in the degenerated beam functions to compute the natural frequencies and modal shapes is limited to 8 terms in each direction because the computing procedure showed numerical instability when using more than 9 terms.

Firstly, the convergence tests were carried out for the results computed by all the methods mentioned above. The responses at the centre of the square plate obtained using the natural frequencies and modes given by the SM are plotted in Fig. 4 for various values of number of terms used in each building block. It may be seen that except for the four term solution, all other results agree well even for non-dimensional time of 100 units, showing that convergence has been reached with only six terms. Figs. 5 and 6 show the response at the same point calculated based on the natural frequencies and modes given by the R-R method in respect of the terms used in the ordinary beam functions and the degenerated beams function respectively. As can be seen from Part (a) of these figures, there are no significant differences between the results for different number of terms for either method, immediately after the plates were released. However, the differences become larger as time increases as can be seen from Part (b) of the figures. While almost no differences are found between the results obtained using 10 and 15 terms in Eq.(13) for the SM, and 6 and 8 terms in each direction of Eq.(1) for the degenerated beam functions, there are obvious disagreements between the results obtained using the ordinary beam functions. For a fair comparison between the convergence rates of the SM and the R-R method with degenerated beam functions, it is useful to note that to get convergence to the same level of agreement, the SM uses a matrix size of $40 \times 40$ where as the R-R method requires about $100 \times 100$. The rates of convergence of the SM and the R-R method with the degenerated beam functions are considerably better than that of the R-R method with the ordinary beam functions. Results of the convergence test are presented only for the square plate, but the same occurrences were observed for plates of other aspect ratios.

Figs 7 shows the transient response of the completely free square plate at (a) the centre, (b) the point $x=0.75 a$ and $y=0.75 b$ and (c) the corner. The natural frequencies and mode shapes used in the computation were obtained using 15 terms in the SM, eight-term degenerated beam functions and 50term ordinary beam functions in the R-R method. Initially, there is an excellent agreement between the results given by all three methods. However, the difference becomes noticeable as time increases. Fig. 8 shows that the response at the corner of the plate at the time of 98 to 100 . While the results obtained based on the SM and the degenerated beam functions are in excellent agreement with each other, there is a large discrepancy between the result based on the ordinary beam functions and others. This is due to the fact that the R-R method using the ordinary free-free beam functions gives higher values for the natural frequencies of the completely free plates than those given by the SM and the R- 
$\mathrm{R}$ method with the degenerated free-free beam functions. Similar differences were also found in the responses at other points of the plate and of the plates for other aspect ratios but are not presented here.

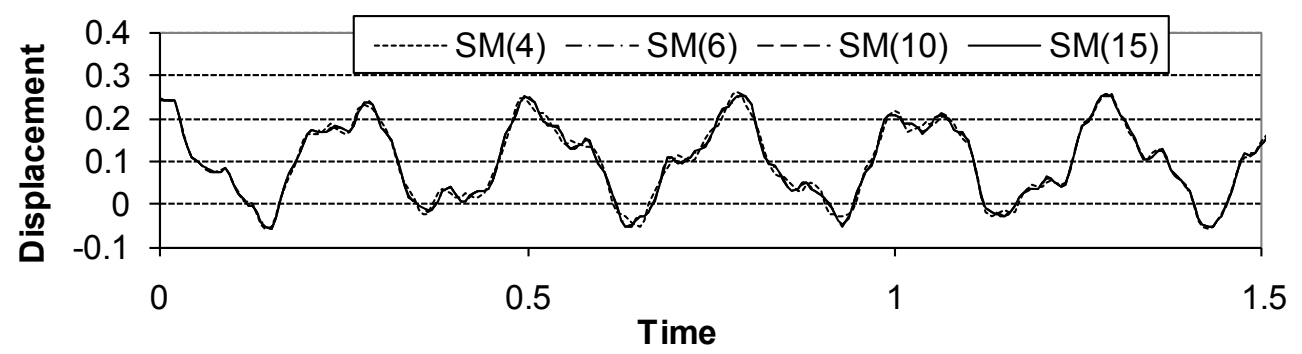

(a)

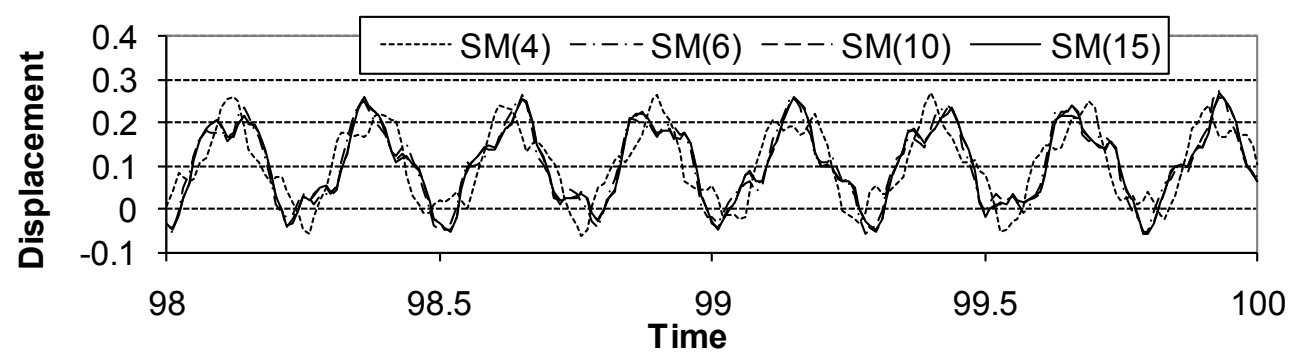

(b)

Fig. 4 The transient response of the completely free square plate at the centre, based on the natural frequencies and modes given by the SM at the time of (a) 0 to 1.5 and (b) 98 to 100.

It can also be seen from Fig. 7 that the plates vibrate about a shifted plane parallel to the original $x y$ plane representing the undeformed state of the plate. The plane shift was also discovered in the responses of the plates for the aspect ratios 1.5 and 2.0. The distance between the planes that are at the centre of vibration and the $x y$ plane almost agree with the displacement contribution of the first mode, which is the rigid body motion in dimensionless distance of 1.0, multiplied by the first transient coefficient of the plates, which is 0.1023 for the square plate (Table 1).

The modal superposition method used in this paper to investigate the transient response of the plate gives an insight into how modes participate in the response, as mentioned in the reference [15]. Fig. 8 shows the proportions of modes participating in the transient responses. Since, in the case studied here, the initial disturbance is symmetric about both centre lines of the plate parallel to $x$ and $y$ axes, only doubly symmetric modes participate. The first three modes for the square plate, four and five modes for the plate with aspect ratios 1.5 and 2.0 respectively, dominate about 90 -percent of the responses. The figures also show that the proportion of higher modes contributing to the transient response of the plates with aspect ratios 1.5 and 2.0 are larger than that of a square plate. 


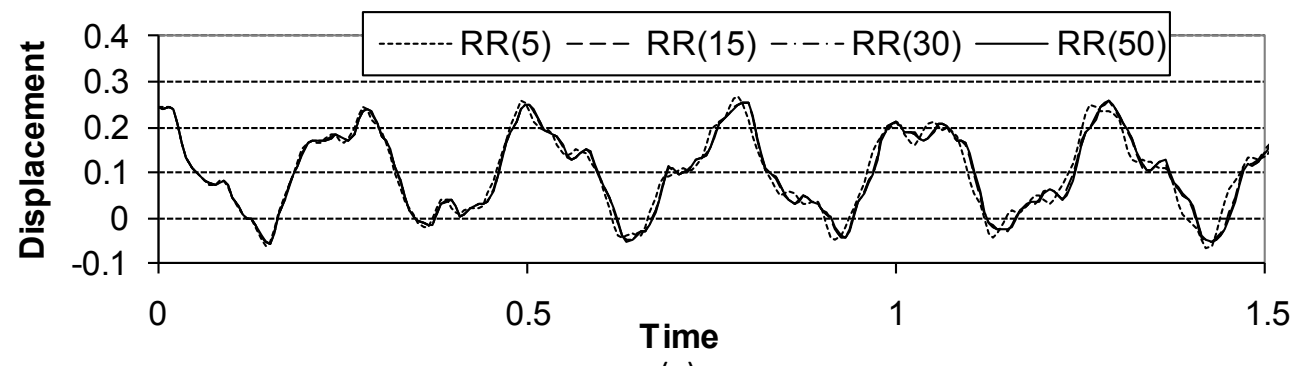

(a)

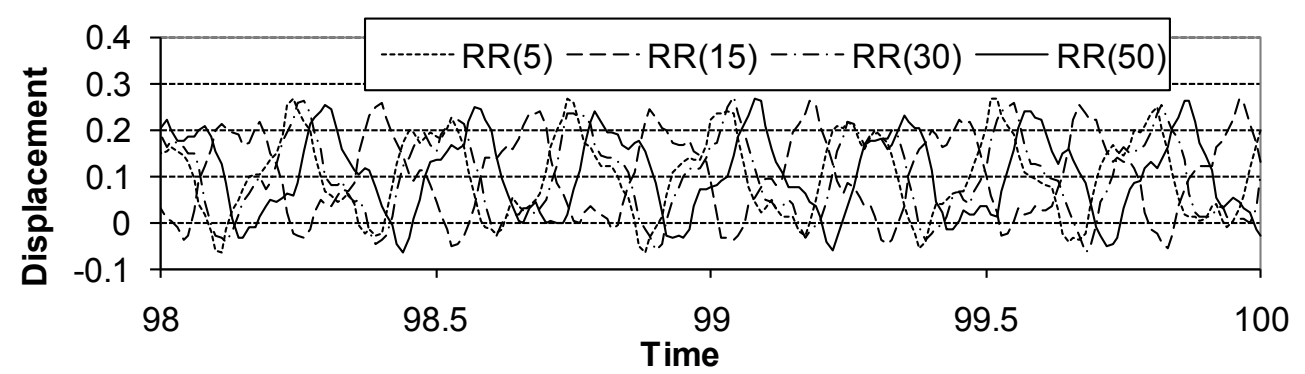

(b)

Fig. 5 The transient response of the completely free square plate at the centre, based on the natural frequencies and modes given by the $R-R$ method with the ordinary beam functions at the time of (a) 0 to 1.5 and (b) 98 to 100.

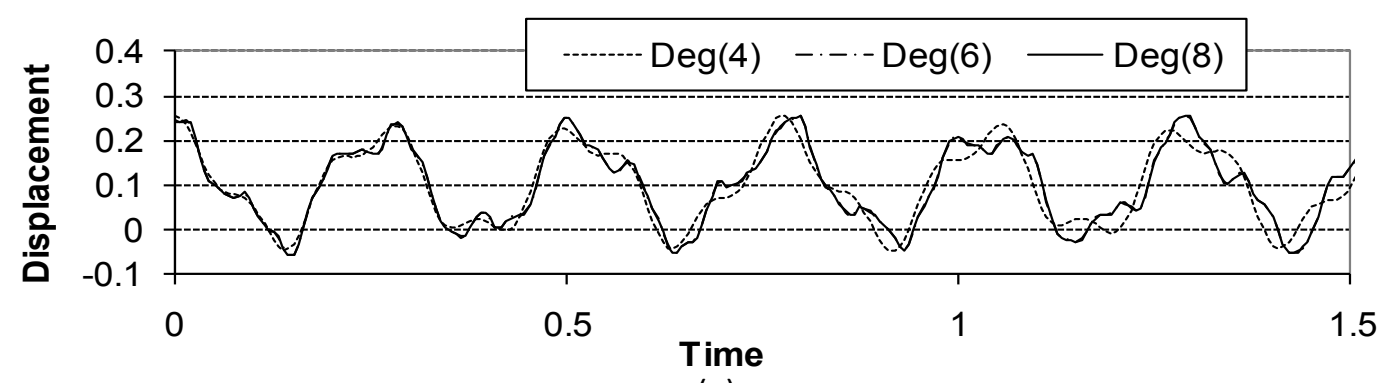

(a)

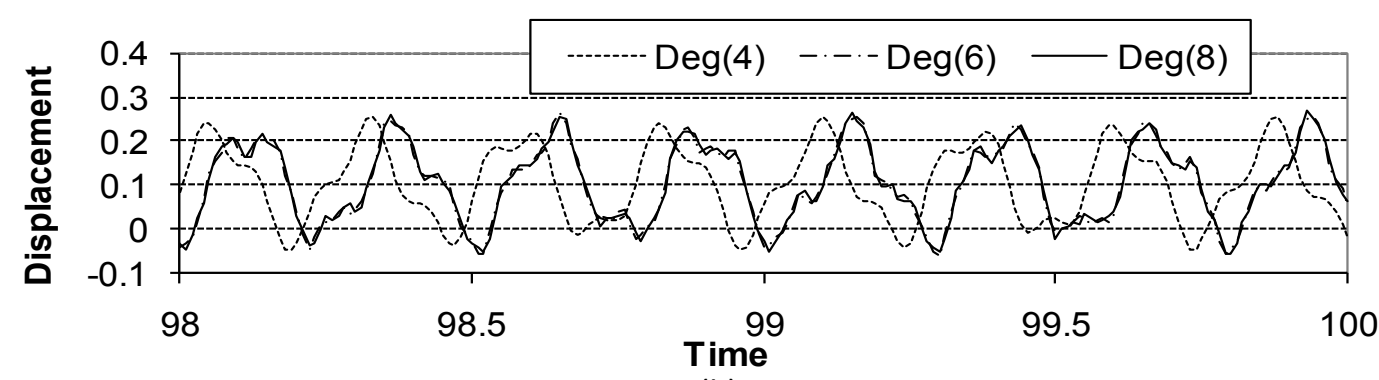

(b)

Fig. 6 The transient response of the completely free square plate at the centre, based on the natural frequencies and modes given by the $R-R$ method with the degenerated beam functions at the time of (a) 0 to 1.5 and (b) 98 to 100 


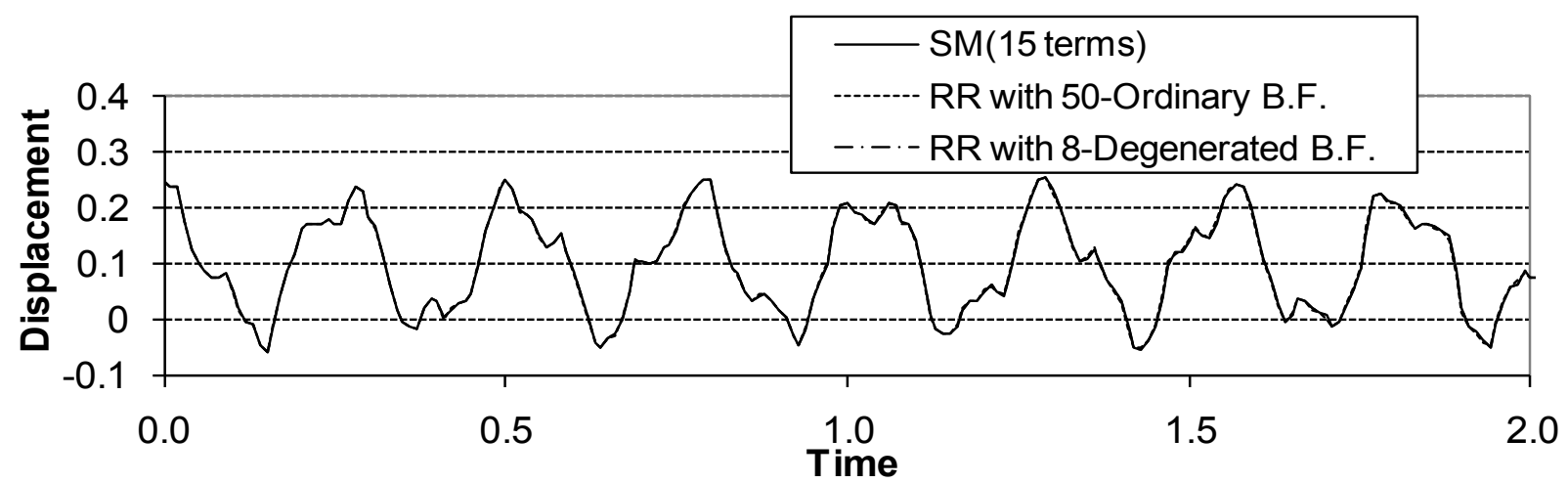

(a)

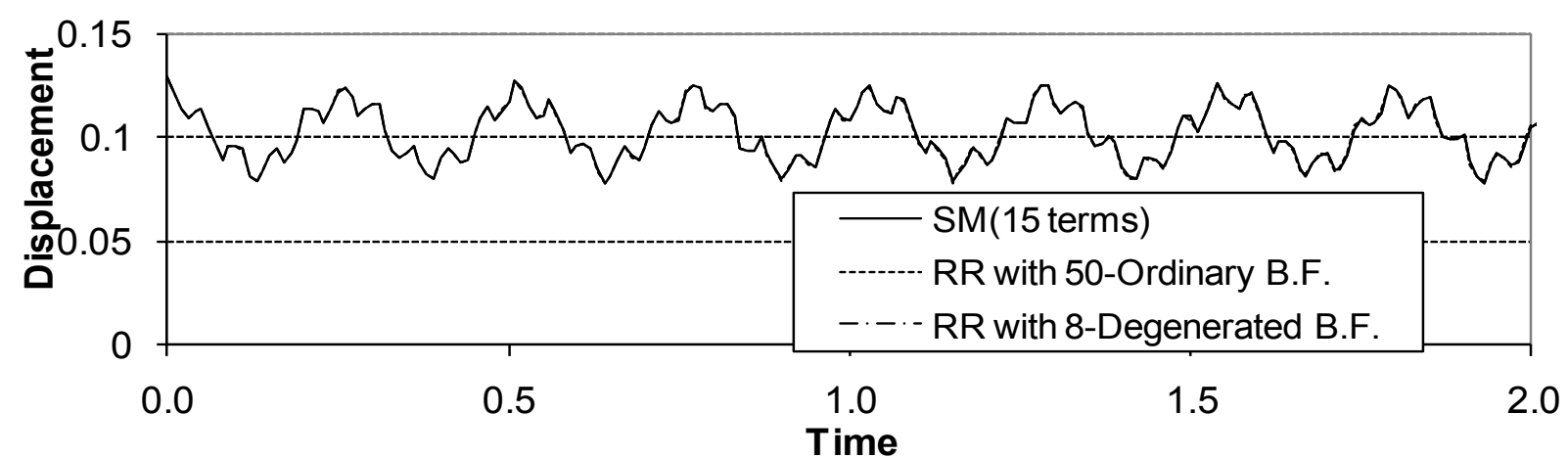

(b)

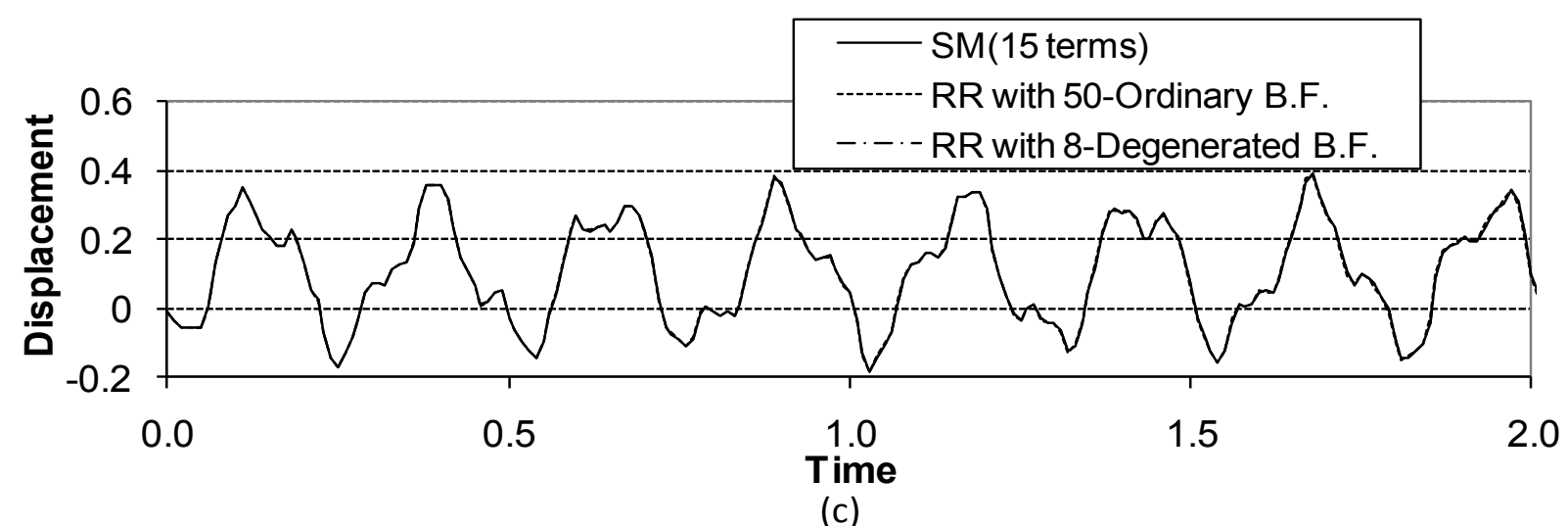

Fig. 7 The transient response of the completely free square plate at (a) the centre, (b) the point $x=0.75 a$ $y=0.75 \mathrm{~b}$ and (c) the corner, obtained using the natural frequencies and modes given by the SM and the R-R method with the ordinary and degenerated beam functions. 


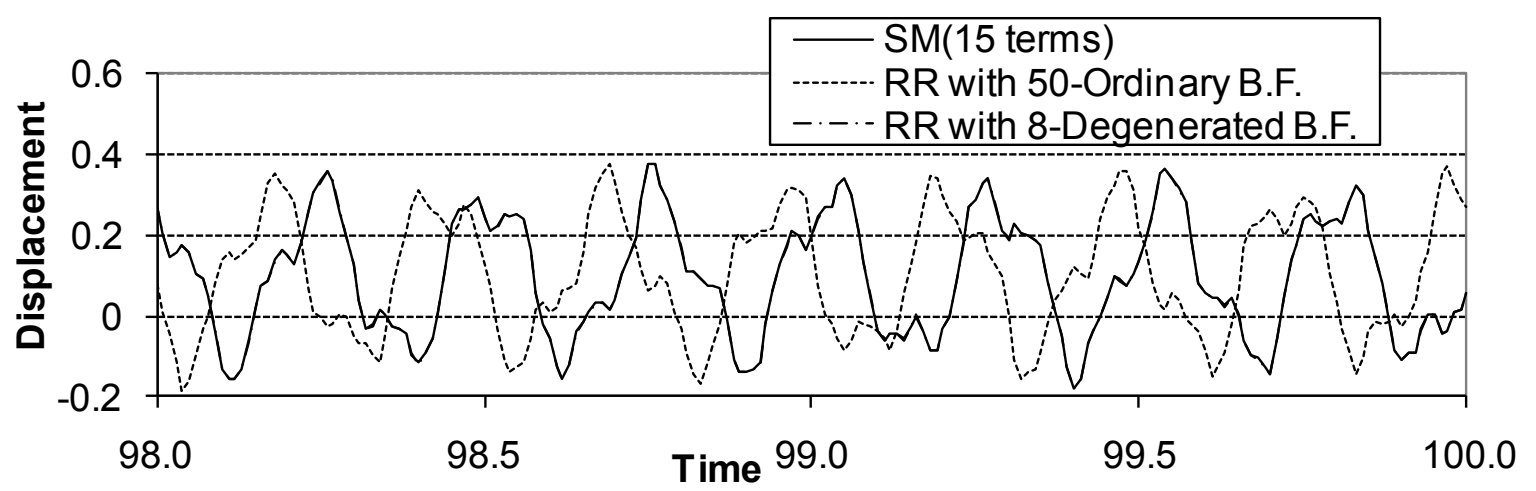

Fig. 8 The transient response of the completely free square plate at the corner at the time of 98 to 100.

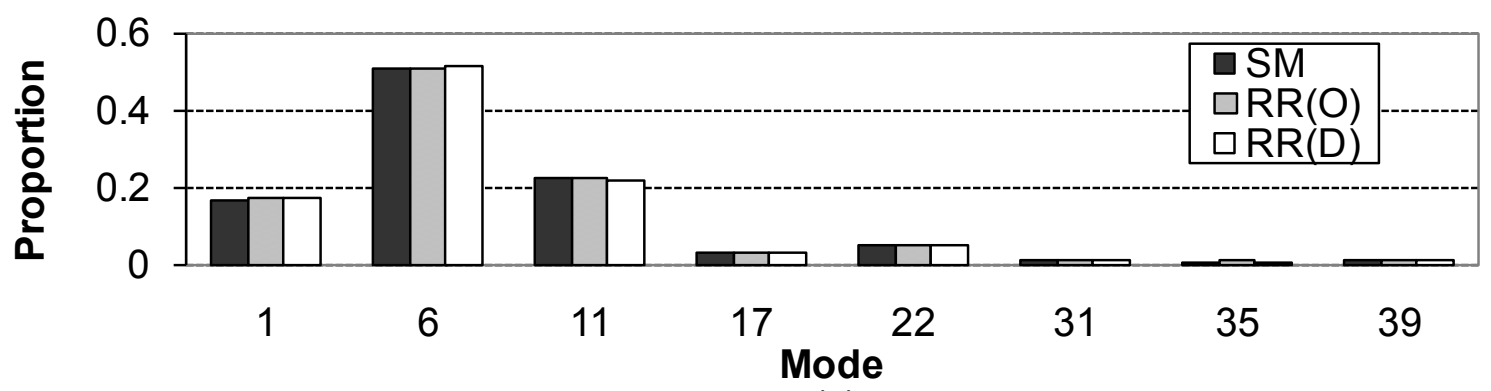

(a)

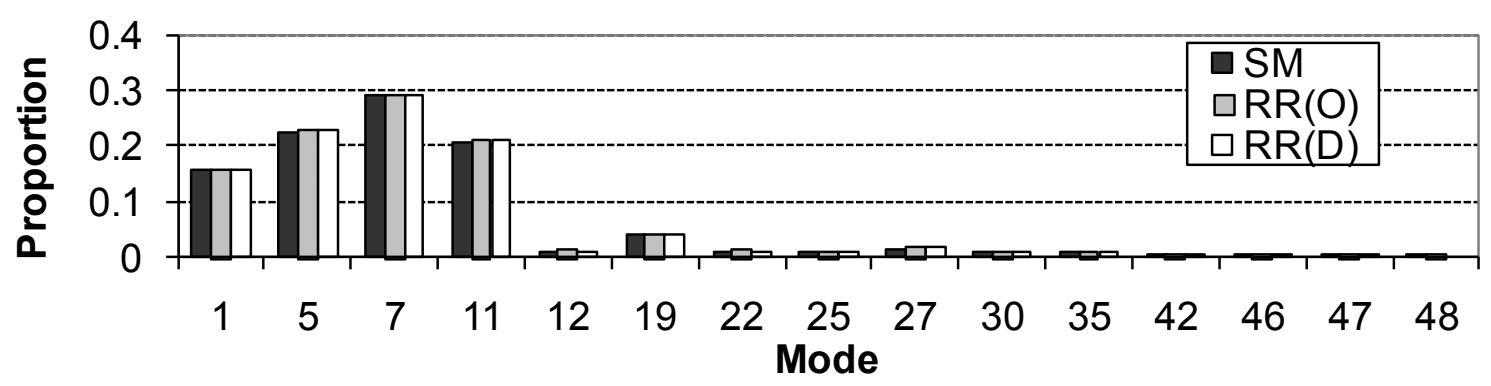

(b)

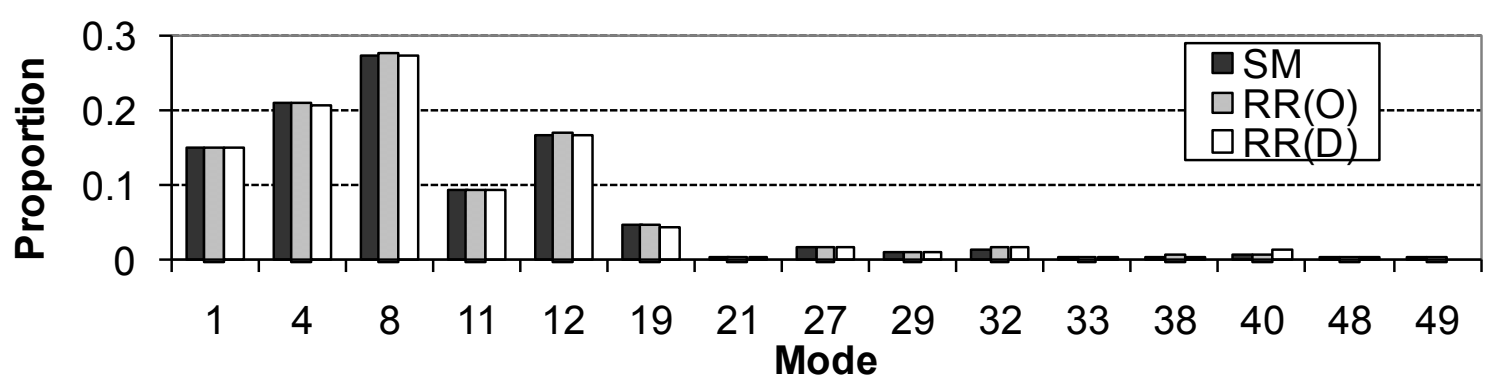

(c)

Fig. 9 The proportion of the participating modes of completely free plates with aspect ratio of (a) 1.0, (b) 1.5 and (c) 2.0 
Table 1 Contribution of participating modes.

\begin{tabular}{|c|c|c|c|c|c|c|}
\hline$\Phi=1.0$ & & & & & & \\
\hline Participating Modes & & 1 & 6 & 11 & & \\
\hline$\lambda^{2}$ & & 0 & 24.27 & 63.69 & & \\
\hline Transient Coefficients & & 0.1023 & 0.8051 & -0.5145 & & \\
\hline Modal displacement & Centre & 1.000 & 0.1433 & -0.0795 & & \\
\hline & $0.75 a, 0.75 b$ & 1.000 & 0.0213 & -0.0031 & & \\
\hline & Corner & 1.000 & -0.2411 & -0.1420 & & \\
\hline$\Phi=1.5$ & & & & & & \\
\hline Participating Modes & & 1 & 5 & 7 & 11 & 12 \\
\hline$\lambda^{2}$ & & 0 & 9.517 & 22.18 & 43.93 & 53.35 \\
\hline Transient Coefficients & & 0.1968 & -1.0559 & -1.3461 & -1.0306 & -0.1060 \\
\hline Modal displacement & Centre & 1.000 & -0.0780 & -0.1032 & -0.0696 & 0.0512 \\
\hline & $0.75 a, 0.75 b$ & 1.000 & -0.0126 & -0.0128 & -0.0090 & -0.0386 \\
\hline & Corner & 1.000 & 0.1333 & 0.1667 & -0.1215 & 0.0838 \\
\hline$\Phi=2.0$ & & & & & & \\
\hline Participating Modes & & 1 & 4 & 8 & 11 & 12 \\
\hline$\lambda^{2}$ & & 0 & 5.366 & 22.00 & 29.68 & 36.04 \\
\hline Transient Coefficients & & 0.2646 & -1.4421 & 1.9101 & 1.3581 & 1.0745 \\
\hline Modal displacement & Centre & 1.000 & -0.0844 & 0.0929 & -0.0255 & 0.0986 \\
\hline & $0.75 a, 0.75 b$ & 1.000 & -0.0139 & 0.0022 & 0.0388 & -0.0129 \\
\hline & Corner & 1.000 & 0.1440 & -0.1244 & -0.0559 & 0.1650 \\
\hline
\end{tabular}

It is known that both the SM and the R-R method give upper bounds for the natural frequencies of completely free plates $[1,4]$ and lowest values would be considered as benchmarks. The SM and the R-R method using the degenerated free-free beam functions give lower values of natural frequencies than those given by the R-R method using the ordinary free-free beam functions for the completely free plates. Those methods also give faster convergence rates. For the same matrix size, however, the SM method gives lower values than those obtained using the degenerated beam functions, and also use of the degenerated beam functions exhibits numerical instability in the results when using more than nine terms in the series. Therefore, it is preferable to use the SM. It seems appropriate at this stage to treat the transient responses obtained using the natural frequencies and modes given by the SM as benchmark results for the response of the plates with all edges simply supported when being suddenly released from all the supports after being subject to an initial displacement corresponding to that due to a uniformly distributed load. The first few participating 
modes, transient coefficients, corresponding natural frequency parameters, $\lambda^{2}[5,6]$ and modal displacements at the centre, the point of $0.75 \mathrm{a}, 0.75 \mathrm{~b}$ and the corner are given in Table 1 . We believe the numerical results would serve as benchmarks for transient response of plates.

\section{Concluding Remarks}

It has been shown that the natural frequencies and modes determined by applying the Superposition Method can be used to calculate the transient response of completely free plates accurately and efficiently. The results agree well with those obtained using the frequencies and modes found by applying the Rayleigh-Ritz Method for short periods of time. The response based on the R-R modes based on ordinary beam functions are slower to converge compared to the corresponding results based on the degenerated beam functions. The results based on the SM prove to be the fastest to converge. This means for a given matrix size, the SM gives more accurate results for the response. The difference between the results found using the SM and the R-R modes increase with time and the use of the degenerated beam functions shows numerical instability during the computation when using more than nine terms in the series. Therefore, the results calculated using the natural frequencies and modes given by the SM are more accurate and reliable, and may be considered as benchmark data for the transient response of the completely free plates.

As expected the transient response is dominated by the lower modes. The plates vibrate about the shifted plane parallel to the original $x y$ plane. The distances between these two planes agree with the displacements for the first mode of the plate (a rigid body translation) multiplied by the first transient coefficient.

In this study, the displacement under uniform load distribution without any excitation is given as the initial condition in order to maintain the focus on transient vibration and keep it simple. Since the procedure used here is valid for various distributions of initial displacement and or initial velocity, it is expected that the response of plates subjected to impulse and other types of general dynamic loading could also be generated using the modes based on the SM. The vibration analysis using the SM modes including a general type of excitation as well as damping effect could be the subject of future work. 


\section{Reference}

[1] A. W. Leissa, Vibration of plates. NASA SP-160 1969.

[2] D. J. Gorman, Free vibration analysis of the completely free rectangular plate by the method of superposition, Journal of Sound and Vibration, 57 (1978), pp. 437-447.

[3] D. J. Gorman, Vibration analysis of plates by the superposition method, Singapore: World Scientific Publishing, 1999.

[4] Y. Mochida, Bounded eigenvalues of fully clamped and completely free rectangular plates, ME Thesis, University of Waikato, 2007.

[5] Y. Mochida and S. Ilanko, Bounded eigenvalues of completely free rectangular plates, Proceedings of the $6^{\text {th }}$ International Symposium on Vibrations of Continuous Systems, Squaw Valley, U.S.A, July, 2007, pp. 22-24.

[6] Y. Mochida and S. Ilanko, Bounded natural frequencies of completely free rectangular plates, Journal of Sound and Vibration, 311 (2008) pp. 1-8.

[7] D. J. Gorman and R. Singhal, Steady-state response of a cantilever plate subjected to harmonic displacement excitation at the base, Journal of Sound and Vibration, 323 (2009) pp. 1003-1015.

[8] E. M. Forsyth and G. B. Warburton, Transient vibration of rectangular plates, Journal Mechanical Engineering Science, 2 (1960) pp. 325-330.

[9] A. Craggs, Transient vibration analysis of linear systems using transition matrices, NASA-CR12371968.

[10] K. Nagaya, Transient response of a continuous plate on elastic supports, Journal of Sound and Vibration, 47 (1976) pp. 359-370.

[11] T. Rock and E. Hinton, Free vibration and transient response of thick and thin plates using the finite element method, Earthquake Engineering and Structural Dynamics, 3 (1974) pp. 51-63.

[12] J. R. Coleby and J. Mazumdar, Non-linear vibrations of elastic plates subjected to transient pressure loading, Journal of Sound and Vibration, 80 (1982) pp. 193-201.

[13] Z. Celep, On the time-response of square plates on unilateral support, Journal of Sound and Vibration, 125 (1988) pp. 305-312.

[14] Y. Nath and K. K. Shukla, Non-linear transient analysis of moderately thick laminated composite plates, Journal of Sound and Vibration, 247 (2001) pp. 509-526.

[15] S. Abrate, Transient response of beams, plates, and shells to impulsive loads, Proceedings of 2007 ASME International Mechanical Engineering Congress and Exposition, Seattle, Washington, USA, November 2007, pp. 107-116.

[16] D. Young, Vibration of rectangular plates by the Ritz method, Journal of Applied Mechanics, 17 (1950) pp. 448-453. 
[17] S. F. Bassily and S. M. Dickinson, On the use of beam functions for problems of plates involving free edges, Journal of Applied Mechanics, 42 (1975), pp. 858-864.

[18] F. W. Williams and W. H. Wittrick, An automatic computational procedure for calculating natural frequencies of skeletal structures, International Journal of Mechanical Sciences, 12 (1970) pp. 781-791.

[19] W. H. Wittrick and F. W. Williams, A general algorithm for computing natural frequencies of elastic structures, Quarterly Journal of Mechanics and Applied Mathematics, 24 (1971) pp. 263-284. [20] W. H. Wittrick and F. W. Williams, An algorithm for computing critical buckling loads of elastic structures, Journal of Structural Mechanics, 1 (1973) pp. 497-518.

[21] S. Timoshenko and S. Woinowsky-Krieger, Theory of plates and shells, Second ed, McGrawHill, Inc., Singapore (1959). 